\title{
Development of a radio-frequency quadrupole cooler for high beam currents
}

\author{
Ramzi Boussaid," G. Ban, G. Quéméner, Y. Merrer, and J. Lorry \\ LPC-IN2P3, ENSICAEN, 6 Boulevard Maréchal Juin, 14050 Caen, France
}

(Received 18 May 2016; published 6 December 2017)

\begin{abstract}
The SHIRaC prototype is a recently developed radio-frequency quadrupole (RFQ) beam cooler with an improved optics design to deliver the required beam quality to a high resolution separator (HRS). For an isobaric separation of isotopes, the HRS demands beams with emittance not exceeding $3 \pi \mathrm{mm} \mathrm{mrad}$ and longitudinal energy spread $\sim 1 \mathrm{eV}$. Simulation studies showed a significant contribution of the buffer gas diffusion, space charge effect and mainly the rf fringe field to degrade the achieved beam quality at the RFQ exit. A miniature rf quadrupole ( $\mu \mathrm{RFQ}$ ) has been implemented at that exit to remove the degrading effects and provide beams with $1 \mathrm{eV}$ of energy spread and around $1.75 \pi \mathrm{mm}$ mrad of emittance for $4 \mathrm{~Pa}$ gas pressure. This solution enables also to transmit more than $60 \%$ of the incoming ions for currents up to $1 \mu \mathrm{A}$. Detailed studies of this development are presented and discussed in this paper. Transport of beams from SHIRaC towards the HRS has been done with an electrostatic quadrupole triplet. Simulations and first experimental tests showed that more than $95 \%$ of ions can reach the HRS. Because SPIRAL-2 beams are of high current and very radioactive, the buffer gas will be highly contaminated. Safe maintenance of the SHIRaC beam line needs exceptional treatment of radioactive contaminants. For that, special vinyl sleep should be mounted on elements to be maintained. A detailed maintenance process will be presented.
\end{abstract}

DOI: 10.1103/PhysRevAccelBeams.20.124701

\section{INTRODUCTION}

In the context of new nuclear facility generation, the SPIRAL-2 project was installed at GANIL laboratory in France [1-3]. Using ISOL production method, rare and exotic ion beams with intensities up to $1 \mu \mathrm{A}$ and emittances up to $80 \pi \mathrm{mm}$ mrad as well as energy spread of some tens of electron volts will be available [4]. These beams will be delivered to low energy experiments of SPIRAL-2 such as desintegration, excitation and storage of ion radioactive (DESIR) [5,6]. Regardless of the high currents SPIRAL-2 beams advantage, the isobaric contaminations [7] will be a major disadvantage for DESIR's typical experiments which require the highest purified beam.

A high resolution separator (HRS) enabling the suppression of undesirable isobaric ions from SPIRAL-2 beams will be installed in front of DESIR [8,9]. Nominal HRS working demands beams with geometric emittance $\leq 3 \pi \mathrm{mm}$ mrad and longitudinal energy spread $\Delta \mathrm{E} \sim 1 \mathrm{eV}$ [8]. For that end, such beams should be cooled before reaching the HRS entrance. The most universal technique providing efficient beam cooling, independently to the ion mass, is the radiofrequency quadrupole cooler (RFQC) [10]. This technique is already used in several projects [11-15]. Existing RFQCs

\footnotetext{
*boussaidramzii@gmail.com

Published by the American Physical Society under the terms of the Creative Commons Attribution 3.0 License. Further distribution of this work must maintain attribution to the author $(s)$ and the published article's title, journal citation, and DOI.
}

can only handle beams with low intensities ( 100 nA) and small emittances $(\sim 10 \pi \mathrm{mm}$ mrad $)$. As SPIRAL-2 typical beams are of higher currents and larger emittances, a new RFQC called SPIRAL-2 high intensity radiofrequency cooler (SHIRaC) has been developed and tested at LPC-Caen laboratory in France [16,17].

Like existing RFQCs, SHIRaC optics system is divided into three sections: injection section, cooling section and extraction section, Fig. 1 [16]. These sections are connected by exchangeable apertures, Fig. 1. They have been designed to ensure an efficient transmission of input beams. To efficiently cool SPIRAL-2 beams, the injection energy that will bring the ions to the cooling section must first be decelerated down to $100 \mathrm{eV}$, placing special demands on the deceleration optics and RFQ design. The deceleration is done in applying the dc electric field along the injection section: the injection plate electrode is at the high voltage (HV) platform relative to a grounded electrode. Additional injection electrodes are used for a fine-tuning of beam transmission.

The cooling section consists of the main RFQC chamber placed at the HV platform. This chamber is filled with helium buffer gas and encloses the radiofrequency quadrupole (RFQ). It is devoted to efficiently trap the injected ions and then progressively cool them in He gas. To guide such ions along the cooling section, the RFQ electrodes are segmented and dc potentials are applied to these segments. The RFQ structure is $700 \mathrm{~mm}$ in length and is separated into 18 segments. The ions reaching the RFQ exit are extracted and then accelerated by a set of electrostatic 


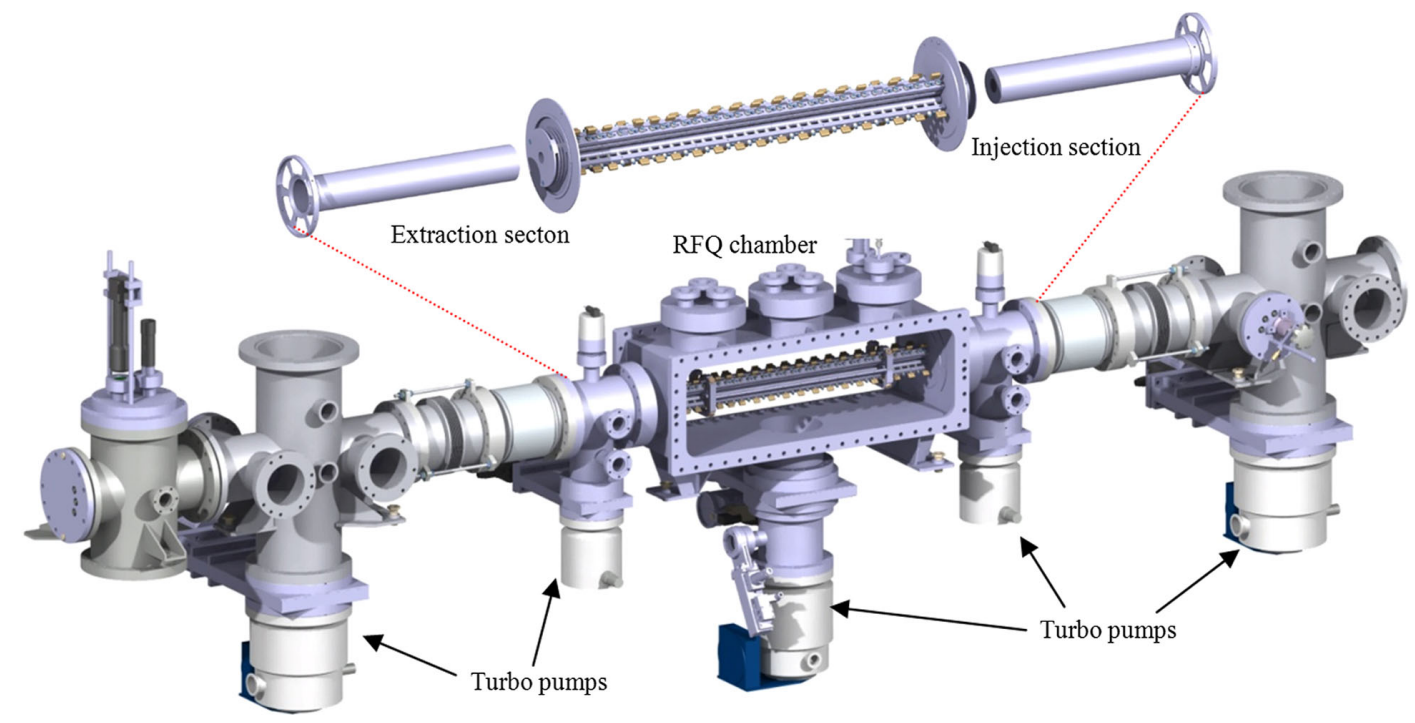

FIG. 1. Schematic of SHIRaC design: 3D layout of SHIRaC beam line with an expanded view of its optics system.

electrodes forming the extraction section. In addition to the improved optics system which captures large beam emittances of some tens of $\pi$ mmmrad, its vacuum system and rf system were also developed [16] in such a way more than $60 \%$ of incoming ions can be transmitted. The vacuum system, based on a differential pumping, allows the reduction of the buffer gas pressures to less than $0.01 \mathrm{~Pa}$ outside the RFQ chamber. With the developed rf system, rf parameters of up to $9 \mathrm{MHz}$ frequency and $9 \mathrm{kV}$ amplitudes can be reached without any breakdown limitations. These rf parameters provide the needed rf confinement field to overcome the space charge effect, due to the high beam currents.

Simulation [17] and experimental studies [16] of a SHIRaC prototype have shown unexpected results. Using rf voltage parameters of $4.5 \mathrm{MHz}$ frequency and $4 \mathrm{kV}$ amplitude (Mathieu parameter $\mathrm{q}=0.4$ ) and $2.5 \mathrm{~Pa}$ buffer gas pressure, this prototype allows handling and cooling beams with current going up to $1 \mu \mathrm{A}$. It provides beams with emittance less than $2.5 \pi \mathrm{mm} \mathrm{mrad}$ and energy spread $\Delta \mathrm{E} \leq 6.5 \mathrm{eV}$ for more than $70 \%$ of transmitted ions. Only the emittance is in agreement with the HRS requirement. The energy spread is, however, much greater than $1 \mathrm{eV}$ and its reduction is mandatory for optimum running of HRS. These studies also showed that the degrading effects (space charge, rf heating and buffer gas diffusion) have considerable impact on the cooling process and thereafter on the beam quality $[16,17]$. Another irrelevant degrading effect may occur at the RFQ exit and acts to grow up the energy spread. A description and analysis of this effect will be investigated in the next section.

Study and development of an engineering technique to reduce this irrelevant effect and then to avoid degradation of the cooled beam quality after the RFQ exit are reported. Additional special considerations to attempt to reach beam quality with $\Delta \mathrm{E} \sim 1 \mathrm{eV}$ and $1 \pi \mathrm{mm}$ mrad emittance with higher ion transmission are investigated.

In order to transport the cooled beam toward the HRS entrance, an electrostatic quadrupole triplet has been developed. More details about the simulation studies and experimental test of the triplet will also be presented.

Protection methods in handling the RFQC beam line in the presence of radioactive contaminants, due to the high intensity of SPIRAL-2 typical ion beams, are illustrated as well.

\section{BEAM QUALITY DEPENDENCY}

After passing through the cooling section, the cooled beams are extracted and then guided with a small acceleration (only few eV) from the RFQ exit to the extraction plate. Once they cross this plate, they are strongly accelerated [17]. The most critical point in acceleration of the beam is the area between the RFQ exit and the extraction plate exit, where the pressure is close to the RFQ pressure [16]. The small energy spread $(\sim 1 \mathrm{eV})$ and emittance $(\sim 1 \pi \mathrm{mm} \mathrm{mrad})$ achieved at the RFQ exit could be partially lost due to undesirable collisions of the cooled ions with the buffer gas atoms. Beam emittance and energy spread growth arises in the absence of a confinement field to overcome the degrading effects as long as the ions' energy is of some electron volts. Comparative simulation results of beam quality [17], at the RFQ exit and the extraction section exit, in function of the beam currents are presented in Fig. 2. The beam quality degradations can be estimated by relying on its relative variation from the RFQ exit to the extraction section exit. Degradations stemming from the buffer gas diffusion correspond to the gap between curves at low beam currents of less than $100 \mathrm{nA}$. They revolve around the emittance and more importantly around the 

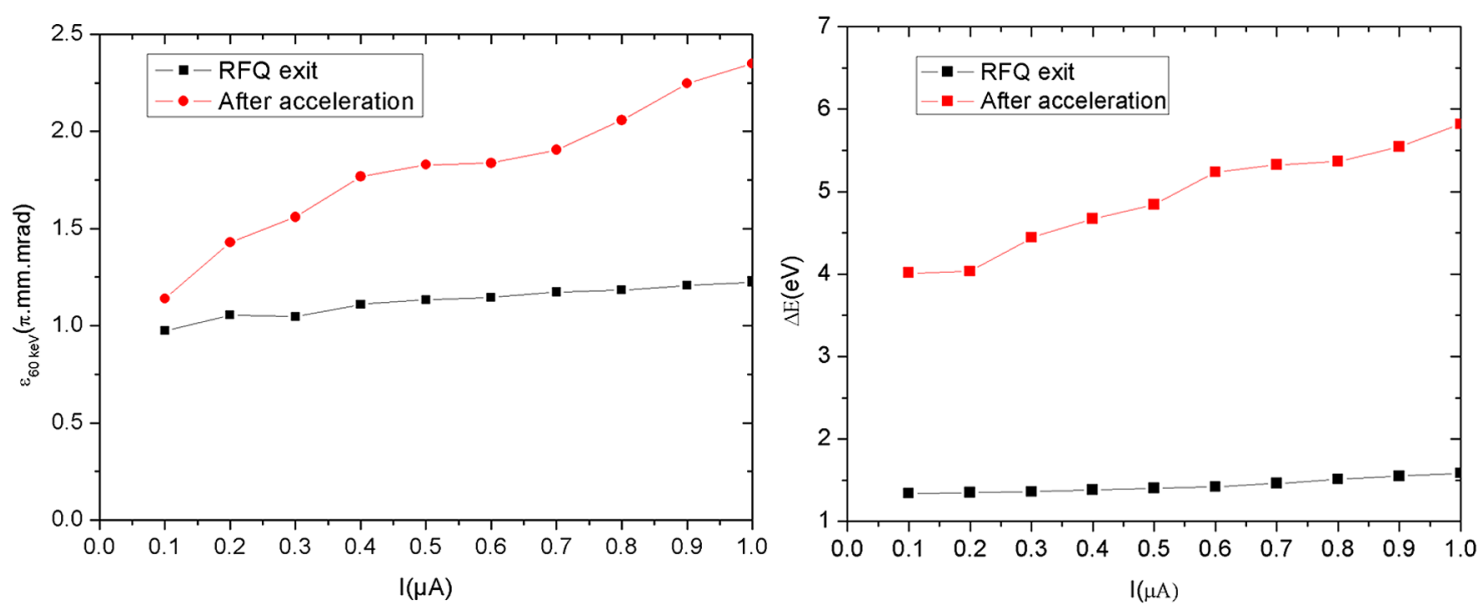

FIG. 2. Space charge effect on the beam quality: (right) simulated results of the beam current effect on the beam emittance; (left) longitudinal energy spread $\Delta \mathrm{E}$ dependency to the beam current [17].

energy spread and are of $0.2 \pi \mathrm{mm} \mathrm{mrad}$ and $2.5 \mathrm{eV}$, respectively. The gap progressively widens with the beam currents while the space charge effect increases with them. The arising of this phenomenon can be observed in the rapid emittance growth with the beam currents (Fig. 2, left). The energy spread is in the same way affected as it increases (Fig. 2, right). This degrading behavior was also observed experimentally, Fig. 3. Regardless of these degradations, a good agreement between simulation and experimental results has been obtained.

Degradation at low beam current $(\leq 100 \mathrm{nA})$, where the space charge effect can be neglected, is expected to be due to the gas diffusion and is of $10 \%$ for the emittance and $\geq 300 \%$ for $\Delta \mathrm{E}$. The energy spread growth is too large to be stemming only from the gas diffusion. On one hand, this is because it is much greater than those provided with the existing cooler, notwithstanding that the gas pressure is at the same level and the vacuum system is not as powerful

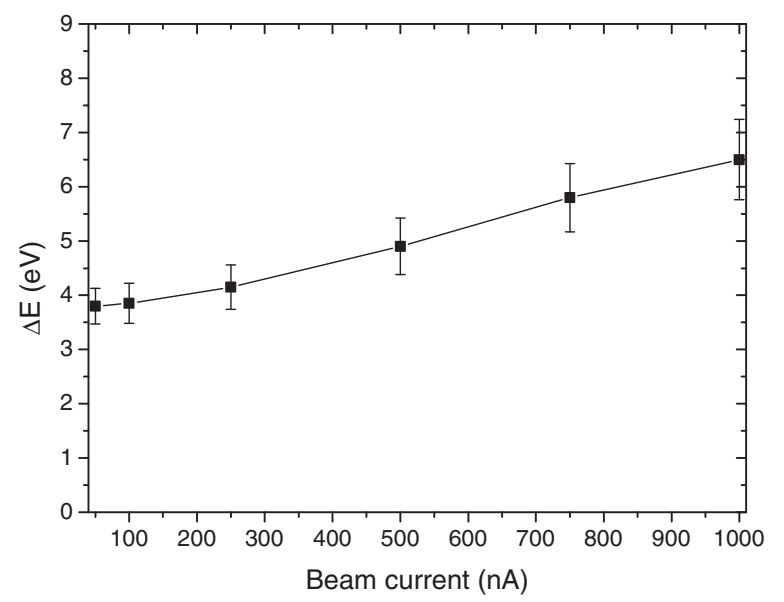

FIG. 3. Space charge effect on the longitudinal energy spread: experimental results of the beam current effect on the longitudinal energy spread at the RFQC exit [16]. as SHIRaC's [13]. On the other hand, it is because the gas diffusion has a small effect on the beam energy that does not exceed $10 \%$.

The main difference between previous RFQCs and SHIRaC lies in the rf voltage parameters used, which were $\leq 1 \mathrm{kV}$ amplitude and $\leq 1 \mathrm{MHz}$ frequency in the former [18], compared to more than $2.5 \mathrm{kV}$ and $4.5 \mathrm{MHz}$ in the latter. This leads us to study the rf parameters effect on the energy spread. In Fig. 4, the variation of the energy spread as a function of the rf voltage amplitudes $V_{\text {rf }}$ for beam current of $1 \mu \mathrm{A}$ is presented. Contrary to the expected behavior of the cooled beam quality with the voltage amplitudes, an increase of the energy spread occurs. This increase is not due to the voltage amplitudes effect on the cooling (i.e., on the energy spread) but rather to the rf fringe field at the RFQ exit, i.e., derivative of the rf voltage amplitude. The rf fringe field gives rise to a longitudinal rf



FIG. 4. Confinement effect of rf voltage amplitude on the longitudinal energy spread: variation of longitudinal energy spread $\Delta \mathrm{E}$ with the Mathieu parameter q (i.e. rf voltage amplitude) [16]. 




FIG. 5. Layout of the developed extraction section and relative positioning of the $\mu$ RFQ from the main RFQ.

field, which acts on the longitudinal velocities distribution of the cooled ions via its randomness characteristic. Thereafter, it results in a degradation of this distribution and, consequently, in an increase of the energy spread.

\section{BEAM QUALITY IMPROVEMENT}

As cited above, the space charge and buffer gas diffusion contribute to the energy spread growth. The largest contribution is due to the rf fringe field. In order to reduce these degrading effects, an optical device providing both the transport of cooled ions from one vacuum stage to another, with only minor disturbances, and a confinement field, should be implemented at the RFQ exit. One option is to set up a miniature RFQ ( $\mu \mathrm{RFQ})$.

\section{A. Miniature rf quadrupole ( $\mu \mathrm{RFQ})$}

The $\mu \mathrm{RFQ}$ device technique was used in several projects [19-21] to only reduce the gas diffusion. In the case of SHIRaC, this device should: (i) overcome the space charge effects occurred at the RFQ exit; (ii) reduce the rf fringe field effect: cutoff of rf voltage derivative propagation; and (iii) guide the cooled ion beam to a region where their energy is of a few tens $\mathrm{eV}$, so then they can resist degrading effects.

Simulation studies of this $\mu \mathrm{RFQ}$ related to the optimization of its design and its position relative to the RFQ exit were conducted. Presented are computer simulations using SIMION 8.1 [22] for dependency of the beam quality on the rf voltage amplitudes $\mathrm{V}_{\mathrm{rf}}$, buffer gas pressure $\mathrm{P}_{\mathrm{RFQ}}$ and beam currents. One thousand ${ }^{133} \mathrm{Cs}^{+}$ions with $60 \mathrm{keV}$ of energy and $80 \pi \mathrm{mm}$ mrad of beam emittance were used. Preliminary simulations showed that the optimized $\mu \mathrm{RFQ}$ dimensions are $40 \mathrm{~mm}$ in length and $2 \mathrm{~mm}$ inner radius. In order to keep the lowest buffer gas diffusion whilst avoiding any breakdown between the $\mu \mathrm{RFQ}$ and the electrodes of the RFQ, the $\mu$ RFQ's electrodes are half cylinder. With this method, the pressure distribution outside the RFQ chamber is reduced around $10 \%$.

For ion stability reasons, the $\mu$ RFQ should operate with the same frequency as the RFQ's. For an optimum cooling and higher ion transmission, it is recommended to operate with the same Mathieu parameter q.

To adequately extract the ions from the $\mu \mathrm{RFQ}$ while avoiding any degrading effect on the obtained beam quality, double extraction electrodes of $3 \mathrm{~mm}$ of inner radius are installed at the $\mu$ RFQ exit (Fig. 5). The first electrode is $1 \mathrm{~mm}$ from the $\mu \mathrm{RFQ}$ exit and $2 \mathrm{~mm}$ from the second electrode.

\section{B. Improvement of energy spread}

Computer simulations of the space charge and RF fringe field effects on the cooling process were performed. The simulation results will be investigated to evaluate the energy spread improvement.

Figure 6 illustrates simulated results of the energy spread as a function of the rf voltage amplitude (i.e., Mathieu parameter q), applied to the main RFQ electrodes. The reduction of the rf fringe field effect is clear while the energy spread does not increase with the rf voltage amplitude. The rf voltage amplitude effect on the cooling is seen in decreasing the energy spread until reaching an

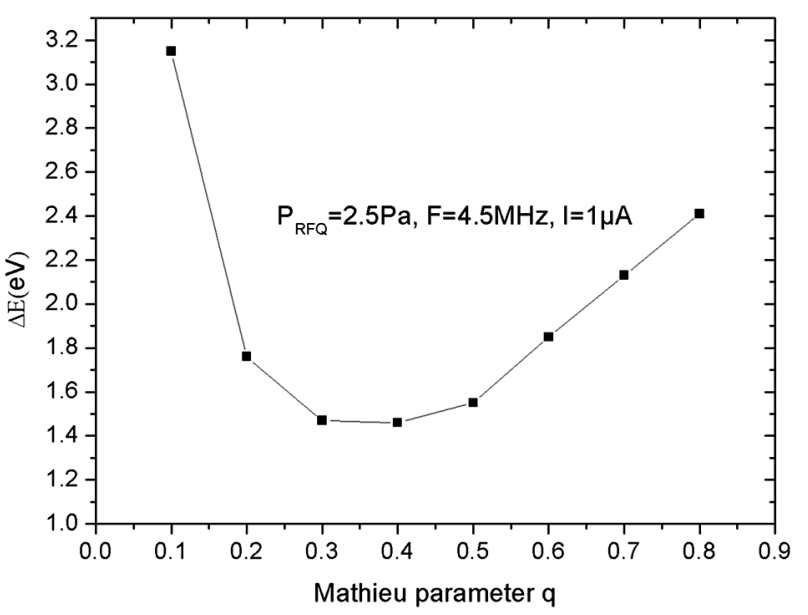

FIG. 6. Effect of the rf voltage amplitude on the longitudinal energy spread $\Delta \mathrm{E}$ for $1 \mu \mathrm{A}$ ion beam: variation of $\Delta \mathrm{E}$ as a function of Mathieu parameter $\mathrm{q}$. 

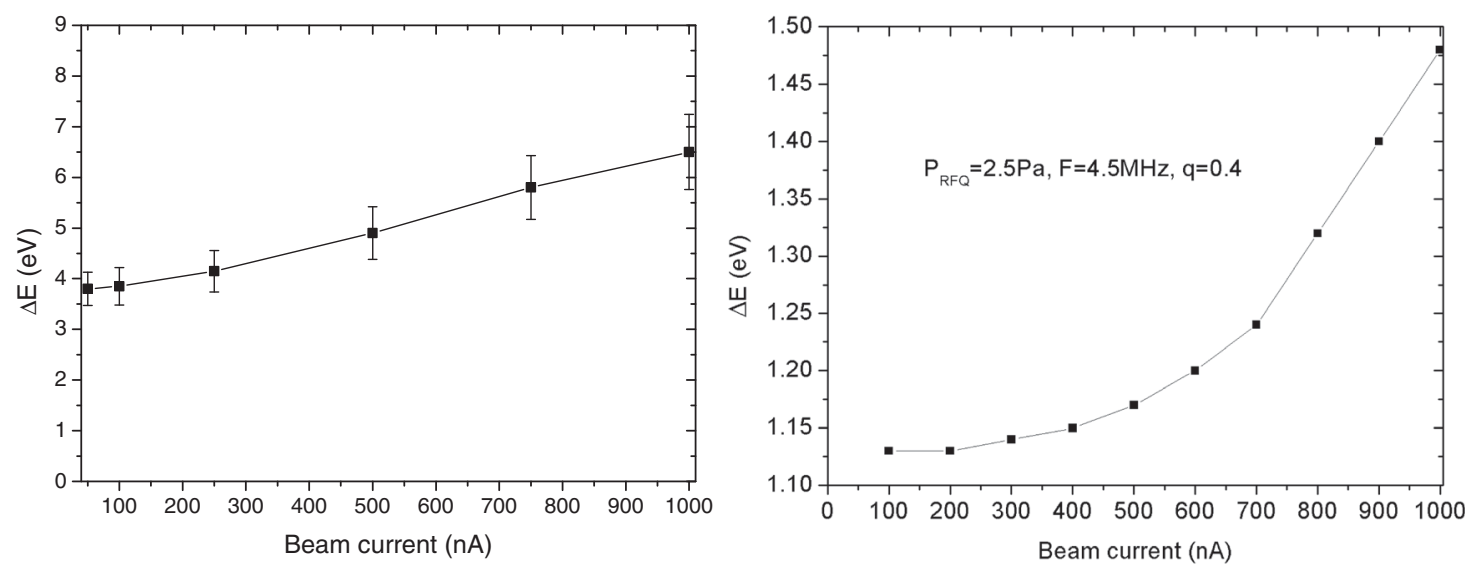

FIG. 7. Illustration of the space charge effect on the longitudinal energy spread with and without $\mu$ RFQ and for Mathieu parameter $\mathrm{q}=0.4$ and $\mathrm{P}_{\mathrm{RFQ}}=2.5 \mathrm{~Pa}$ : Variation of $\Delta \mathrm{E}$ as a function of the beam current without $\mu \mathrm{RFQ}$ (left), variation of $\Delta \mathrm{E}$ as a function of the beam current with $\mu$ RFQ (right).

optimum cooling corresponding to a plateau for q between 0.3 and 0.5 . This behavior reveals the expected rf voltage amplitude effect on the confinement power and thereafter on the beam cooling. The increase in the energy spread for $\mathrm{q}>0.5$ is explained by the apparition of the rf heating effect on the cooling. In addition, we note that the buffer gas diffusion degrading contribution is reduced in view of small energy spread.

The $\mu \mathrm{RFQ}$ contribution to reduce the space charge effect on the energy spread should be noticeable in view of the presence of a confinement field to overcome this effect as long as the cooled ions are slowed down at the RFQ exit. This is clear as the energy spread increases little by little from 1.1 to $1.45 \mathrm{eV}$ with beam currents going up to $1 \mu \mathrm{A}$ (Fig. 7, left). This is also visibly manifested in the reduction of the energy spread growing gap for beam currents ranging from $50 \mathrm{nA}$ up to $1 \mu \mathrm{A}$, which was more than $2.5 \mathrm{eV}$ without the $\mu \mathrm{RFQ}$ [17] versus only $0.3 \mathrm{eV}$.

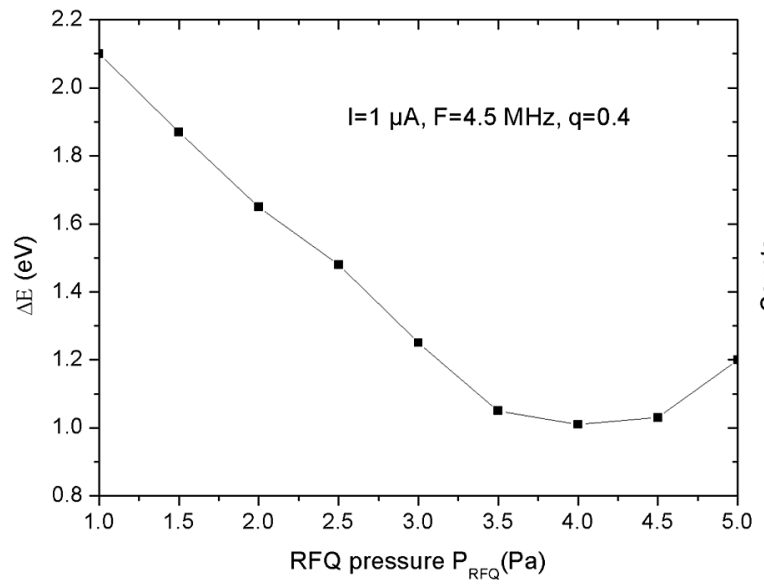

\section{Improvement of beam optics quality}

As shown above, the $\mu$ RFQ contribution is efficient to significantly reduce the degrading effects on the beam quality. Simulations will be performed to further improve the optimum cooling. The beam cooling can be improved in increasing the buffer gas pressure while maintaining low buffer gas diffusion. Thus, any increase of RFQ pressure results in enhancing the cooled beam quality. Figure 8 illustrates this phenomenon as the energy spread minimum, $\Delta \mathrm{E} \sim 1.01 \mathrm{eV}$, is obtained with $4 \mathrm{~Pa}$ of pressure instead of $1.45 \mathrm{eV}$ with $2.5 \mathrm{~Pa}$ without the $\mu \mathrm{RFQ}$. As is expected, the energy spread is improved with the buffer gas pressure until reaching its optimum value at $4 \mathrm{~Pa}$ where the optimum cooling is. The increase in the energy spread at high pressures, beyond $4.5 \mathrm{~Pa}$, appeared to come from the buffer gas diffusion effect at the $\mu$ RFQ exit.

The cooling improvement with the buffer gas pressure should automatically result in reduction of the optimum emittance from $2.5 \pi \mathrm{mm}$ mrad without the $\mu \mathrm{RFQ}$ [16] to

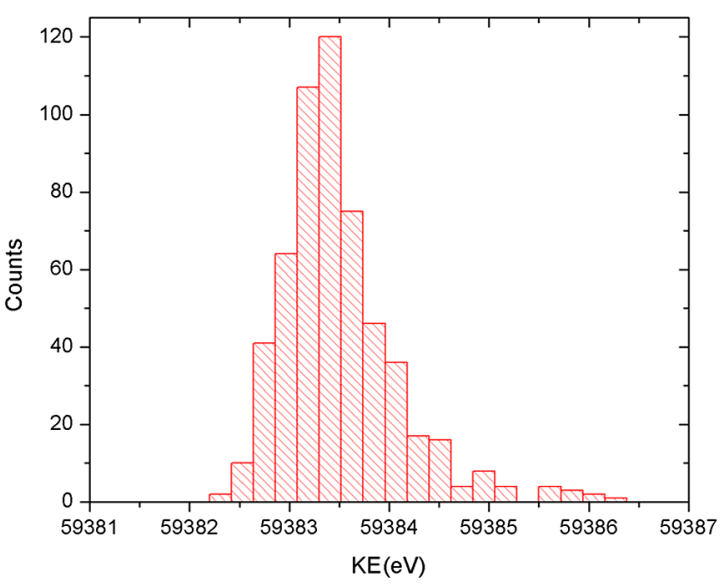

FIG. 8. Effect of RFQ pressure $\mathrm{P}_{\mathrm{RFQ}}$ on the longitudinal energy spread $\Delta \mathrm{E}$ for $1 \mu \mathrm{A}$ of beam current and $\mathrm{q}=0.4$ : variation of $\Delta \mathrm{E}$ as a function of $\mathrm{P}_{\mathrm{RFQ}}$ (left), beam energy histogram taken at $4 \mathrm{~Pa}$ of $\mathrm{P}_{\mathrm{RFQ}}$ (right). 

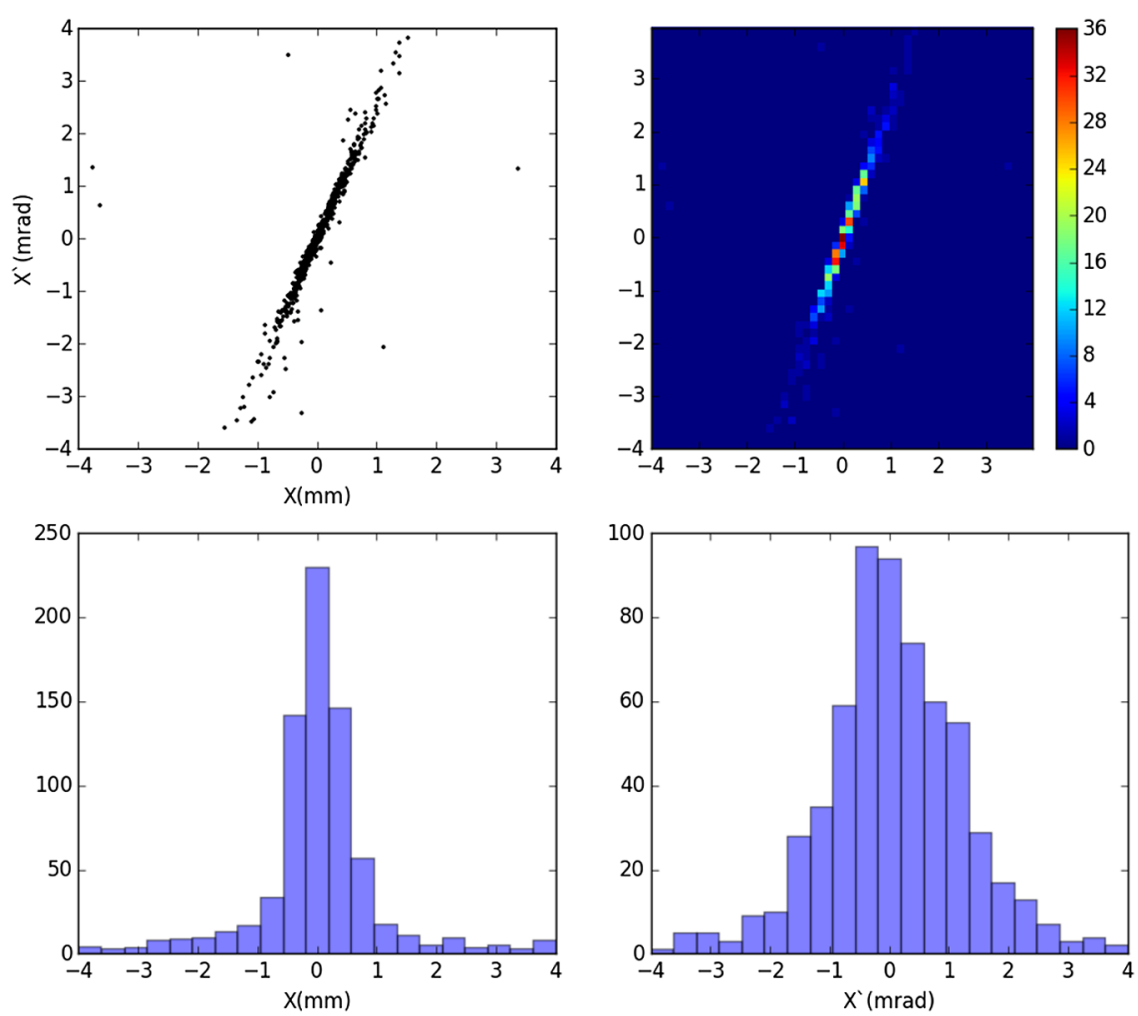

FIG. 9. Diagrams of geometrical beam emittance for cooled beam with $1 \mu \mathrm{A}$ beam current and $4 \mathrm{~Pa} \mathrm{P}_{\mathrm{RFQ}}: \mathrm{X}-\mathrm{X}^{\prime}$ phase space distribution (top), transversal $\mathrm{X}$-axis histogram plot (bottom left), transversal $\mathrm{X}^{\prime}$-axis histogram plot (bottom right).

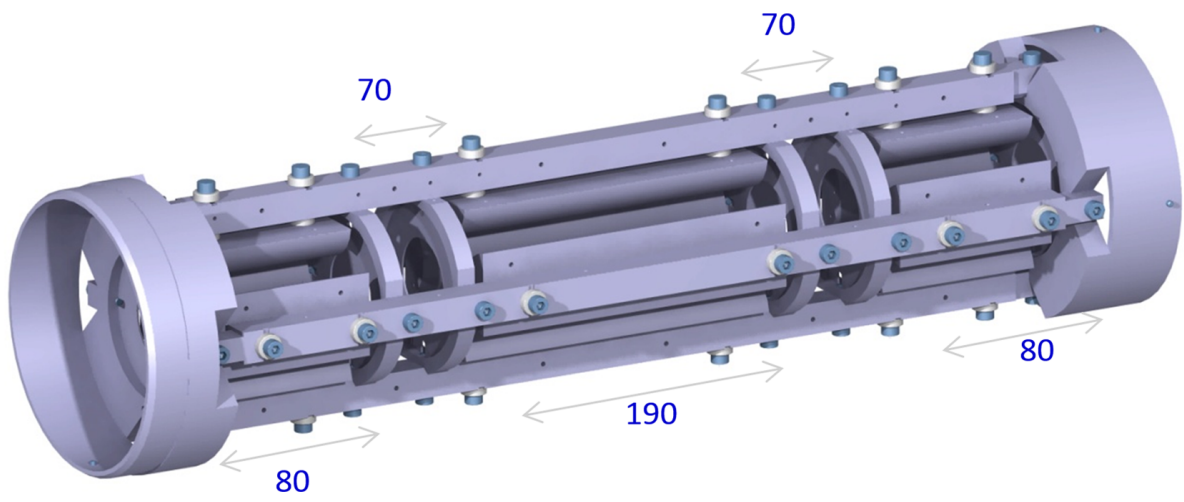

FIG. 10. Schematic view of the electrostatic quadrupole triplet: three quadrupoles with collimator in each quadrupole's side.

around $1.75 \pi \mathrm{mm}$ mrad with respectively 2.5 and $4 \mathrm{~Pa}$. Such beam emittance reduction is shown in Fig. 9. It is also important to note that the simulations showed transmissions remain unchangeable in implementing the $\mu \mathrm{RFQ}$ and exceed $70 \%$ for beam current going up to $1 \mu \mathrm{A}$.

\section{COUPLING RFQ-HRS: QUADRUPOLE TRIPLET}

The ions leaving SHIRaC are accelerated toward an HRS entrance [8] where they should pass through a $1 \times 4 \mathrm{~mm}^{2}$ rectangular slit. Without any ion optical element, the ion
TABLE I. Specification of the electric quadrupole triplet.

\begin{tabular}{lc}
\hline \hline Compounds & Dimensions (mm) \\
\hline 1st quadrupole (EQ 1) & 80 \\
Drift: EQ-EQ & 60 \\
2nd quadrupole (EQ 2) & 160 \\
3rd quadrupole (EQ 3) & 80 \\
Drift: EQ 3-HRS entrance & 230 \\
Total length & 440 \\
Inner radius & 15 \\
Length of collimator & 2 \\
Inner radius of collimator & 15 \\
\hline \hline
\end{tabular}



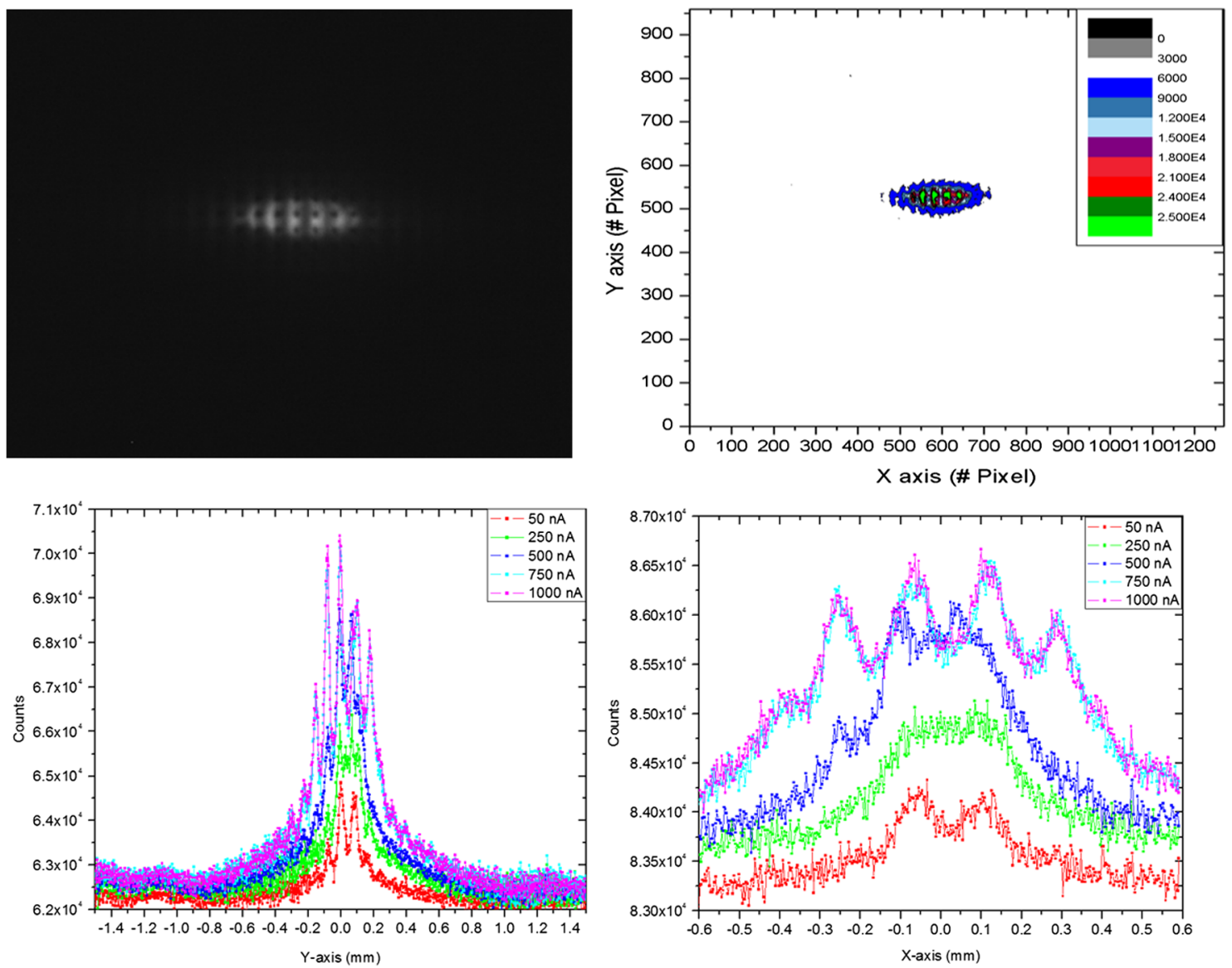

FIG. 11. Beam profile along the transverse axis for various beam currents and with the optimum cooling condition mentioned above: The image represents the transverse beam profile obtained via a CCD camera (top left); contour plot of the transverse beam profile obtained by an RGB analysis method (top right); projection of the image intensity according to the transverse axis for various beam currents (bottom).

losses are significant and less than $20 \%$ of them can reach the HRS. In order to avoid these losses, the element should be installed between the extraction section exit and the HRS entrance and must allow a focusing of the beam at that slit. It also must be run with only a few kilovolts of voltage dc (less than $5 \mathrm{kV}$ ) for energy beams up to $60 \mathrm{keV}$. The suitable solution is a multiplet of electrostatic quadrupole which can provide stronger focus than the Einzel lens [23].

Because the tuning of a quadrupole triplet is much easier than other quadrupole multiplets and a triplet remains flexible enough to satisfy various requirements in the first order focusing properties, a quadrupole triplet has been adopted. Design of the triplet and the optimum dimensions of the quadrupoles were done by Cosy Infinity program [24]. The optimum design is shown in Fig. 10 and their dimensions are illustrated in Table I. Simulations have shown important aberrations of the beam through the triplet, due to the edge effects of their electrodes. In order to reduce them, collimators were installed on both sides of each quadrupole. The voltages are applied to the triplet in such a way that, in both $\mathrm{x}-\mathrm{z}$ and $\mathrm{y}-\mathrm{z}$ transverse planes, converging and diverging lenses alternate. The $\mathrm{y}-\mathrm{z}$ plane has been taken to be the diverging-converging-diverging plane and $\mathrm{x}-\mathrm{z}$ plane to be the converging-diverging-converging plane. The optimum voltages for a beam of $5 \mathrm{keV}$ are of 130, 220 and $130 \mathrm{~V}$ respectively for the first, second and third quadrupoles. However, for a beam of $60 \mathrm{keV}$ they are of $2.3,4$ and $2.3 \mathrm{kV}$ respectively.

In the below figure are the measurements of the transverse beam profile distributions along the transverse axis for various beam currents. The distributions consist of an image intensity's projection along such an axis of the

TABLE II. The full width FWHM of Gaussian fits of the cooled beam transverse profiles for various beam currents.

\begin{tabular}{lcc}
\hline \hline Intensity $(\mathrm{nA})$ & $\sigma_{\mathrm{x}}(\mathrm{nm})$ & $\sigma_{\mathrm{y}}(\mathrm{nm})$ \\
\hline 50 & 0.231 & 0.347 \\
250 & 0.246 & 0.350 \\
500 & 0.242 & 0.362 \\
750 & 0.308 & 0.579 \\
1000 & 0.374 & 0.586 \\
\hline \hline
\end{tabular}




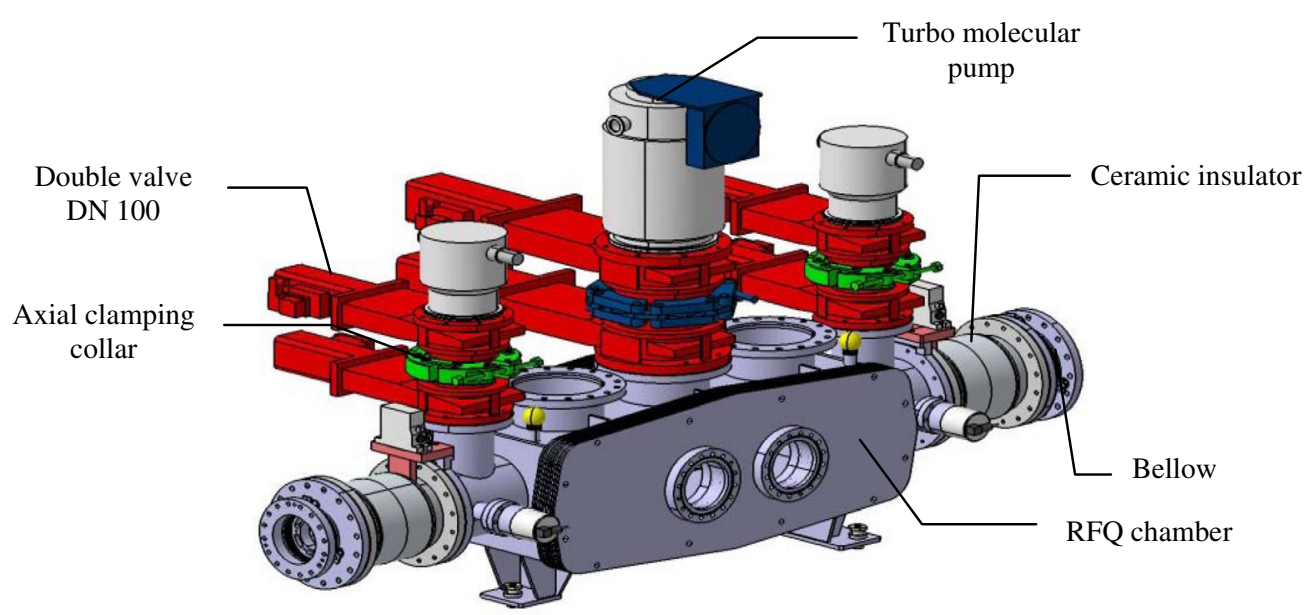

FIG. 12. Protection process of the turbomolecular pumps from radioactive gaseous waste.

transverse beam profile. The beamlets leaving the pepper pot mask of the emittance meter [16] and then in hitting the phosphor screen create the light beams. The latter are transported to a CCD camera. The obtained image is acquired and then analyzed as a transverse beam profile. The image presented in Fig. 11 illustrates an example of beam transverse profile for $1 \mu \mathrm{A}$ beam current and with optimum running conditions. More measurements are taken to study the space charge effect on the transverse beam size for currents range 50 to $1 \mu \mathrm{A}$. Widening of these profiles due to the repulsive space charge effect has been carried out on Fig. 11. To quantitatively study this phenomenon, the full width $\left(\sigma_{\mathrm{x}}\right.$ or $\left.\sigma_{\mathrm{y}}\right)$ of the Gaussian fit of these profiles was determined, Table II. For beam

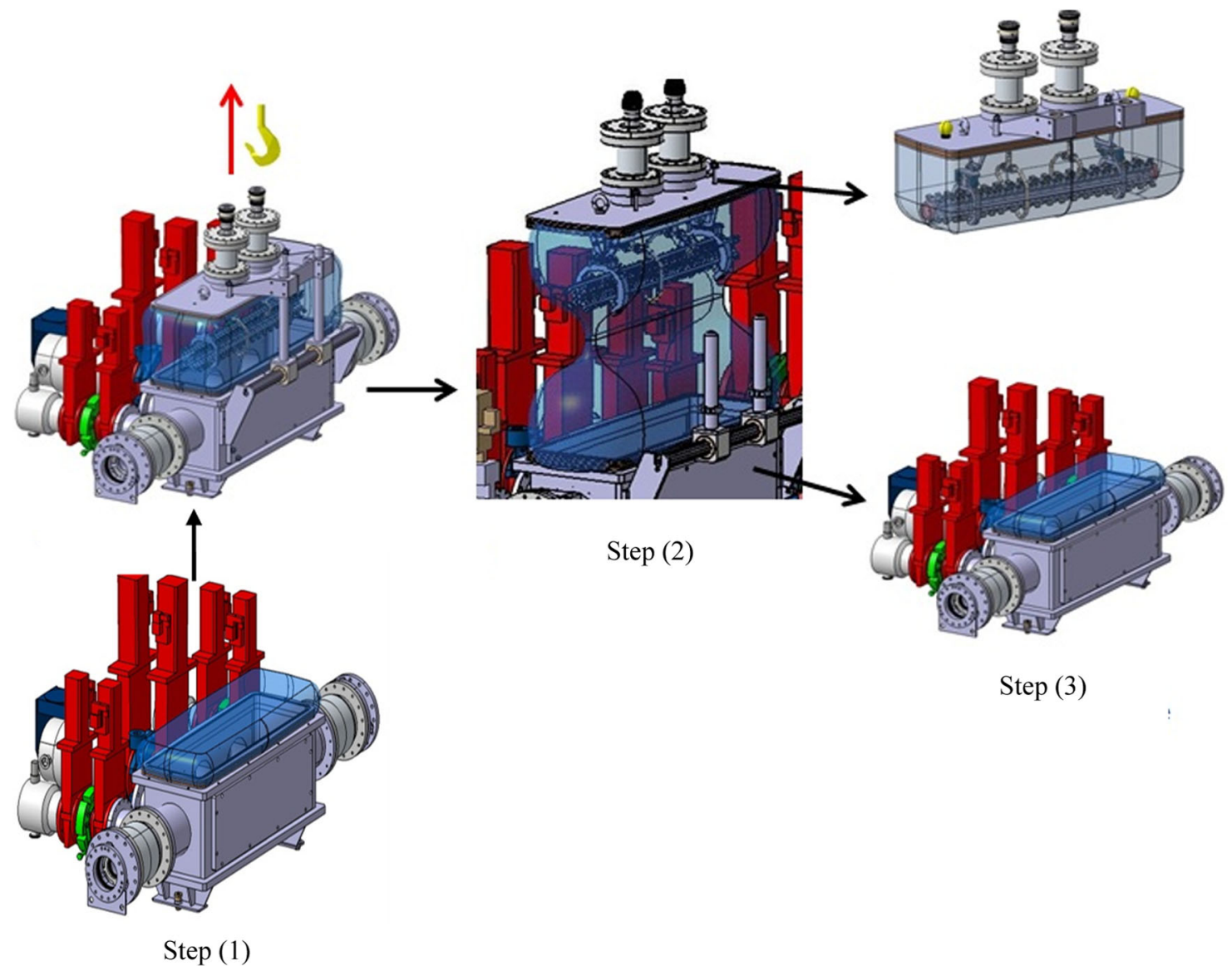

FIG. 13. Safety maintenance of the RFQ module: Establishment of confinement vinyl and withdraw of the module (Step 1), creation of two volumes (Step 2) and separation of two subsets (Step 3). 

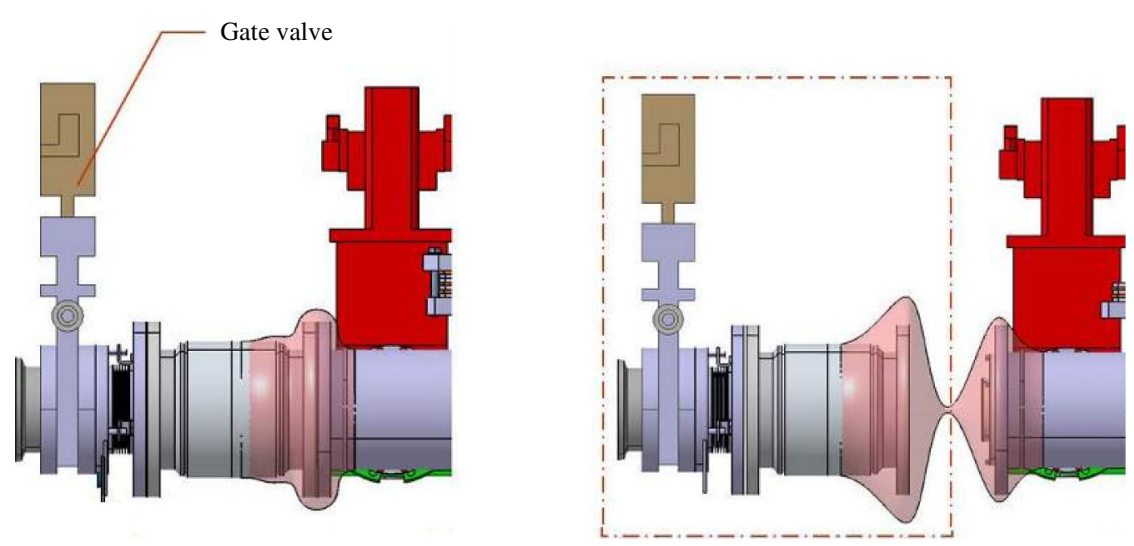

FIG. 14. Technical processes for safety withdraw of elements from the device beam line: setup of the vinyl at the extremity of the insulator (left); separation of volumes by welding the vinyl (right).

currents going up to $1 \mu \mathrm{A}$, the triple of the FWHM $\left(3 \sigma_{\mathrm{x}}\right.$ or $\left.3 \sigma_{\mathrm{y}}\right)$ did not exceed the dimension of the slit $\left(3 \sigma_{\mathrm{x}}<1 \mathrm{~nm} \mathrm{~mm}\right.$ and $\left.3 \sigma_{\mathrm{y}}<4 \mathrm{~mm}\right)$. Therefore, more than 95\% of cooled beams can pass through the slit toward the HRS.

\section{TREATMENT OF RADIOACTIVE CONTAMINANTS}

As mentioned above, SHIRaC will be implemented into the SPIRAL-2 facility and will receive their beams. Because these beams will be radioactive and high currents up to $1 \mu \mathrm{A}$, the buffer gas filling up the RFQ chamber as well as the residual gas existing along the device beam line will be potentially contaminated with radioactivity. The high efficient differential pumping system used for gas pumping [17] is unable to totally remove such gaseous contaminants from the beam line. Particular processes have to be integrated for a safe maintenance of SHIRaC elements, mostly of the RFQ module. As limited maintenance time is required, the processes consist of confining such waste during the maintenance, Fig. 12. Furthermore, it is necessary to protect the turbomolecular pumps from the radioactive waste during maintenance.

During SHIRaC beam-line maintenance, it is necessary to establish continuous confinement of the radioactive waste by mounting a vinyl sleeve, Fig. 13. The below figure illustrates the withdraw step of the RFQ module. First, the RFQ support is vertically extracted once the vinyl sleeve is mounted around this support via maintaining rushes. Then, the volume under the vinyl is separated into two distinct subsets by welding. Finally, the RFQ can be safety repaired while the rest of the elements are enclosed by vinyl sleeve. To reinstall the RFQ support, we should first set up the vinyl sleeve and then withdraw it before mounting the RFQ.

The same process has to be used for any element of the SHIRaC beam line. For example, the withdraw process of the ceramic insulator is presented in Fig. 14. A vinyl sleeve is mounted on the extremity of such insulator. Then, separated volumes under vinyl are obtained by welding the vinyl.

Furthermore, to protect the turbopumps, it is necessary to mount two double valves with an axial clamping collar on each pump. The first valve is fixed to the pump module and the second to the beam line. The sweep of gussets between the double valves is performed before disconnection of the module. The gas recovered following this sweep is analyzed and, if necessary, stored for radioactive decay. The recycling of the gas can also be included with an improved vacuum system.

\section{CONCLUSION AND PERSPECTIVES}

The ingenious solution of the $\mu \mathrm{RFQ}$ showed high efficiency in improving the beam quality by reducing the beam emittance and the energy spread. The achieved beam quality matches the expected values of SHIRaC requirements and thereafter an isobaric purification with the HRS will be possible.

The proposed solutions for the treatment of the radioactive gaseous waste and the integration of the $\mu \mathrm{RFQ}$ presented in this paper are under realization.

Owing to these studies, the SHIRaC prototype will provide high beam quality for currents never yet handled. However, the cooling of beams with currents exceeding $1 \mu \mathrm{A}$ will need a new RFQ cooler design and even the $\mu$ RFQ will not be able to overcome the degrading effects. Special considerations of the extraction section should be studied for the next generation of the RFQ cooler for the EURISOL project $[2,25]$ where the beam current will reach $10 \mu \mathrm{A}$.

\section{ACKNOWLEDGMENTS}

We would like to thank the LPC-Trap team for continuous encouragement and support. The vacuum system and mechanical design at LPC Caen (France) are gratefully acknowledged for their kind assistance in the development of the project. 
[1] Letter of intent for SPIRAL 2.

[2] M. Lewitowicz, The SPIRAL 2 project, Nucl. Phys. A805, 519 (2008).

[3] M. Lewitowicz, Status of the SPIRAL2 project, Acta Phys. Pol. B 42, 877 (2011).

[4] S. Gales, SPIRAL 2 at GANIL: Next generation of ISOL facility for intense secondary radioactive ion beams, Nucl. Phys. A834, 717c (2010).

[5] The DESIR facility, letter of intents for SPIRAL 2, 2006.

[6] B. Blanc et al., DESIR: The SPIRAL2 low-energy beam facility, technical proposal for SPIRAL2 instrumentation, 2008, pp. 1-102.

[7] E. M. Ramirez, S. Cabaret, and D. Lunney, The ion circus: A novel circular Paul trap to resolve isobaric contamination, Nucl. Instrum. Methods Phys. Res., Sect. B 266, 4460 (2008).

[8] D. Toprek et al., DESIR high resolution separator at GANIL (France), Nucl. Technol. Radiat. Prot. 27, 346 (2012).

[9] T. Kurtukian-Nieto et al., SPIRAL2/DESIR high resolution mass separator, Nucl. Instrum. Methods Phys. Res., Sect. B 317, 284 (2013).

[10] R. B. Moore, and O. Gianfrancesco, Improving isotope separator performance by beam cooling, Nucl. Instrum. Methods Phys. Res., Sect. B 204, 557 (2003).

[11] G. Bollen, and S. Schwarz, Penning trap mass measurements on rare isotopes status and new developments, J. Phys. B 36, 941 (2003).

[12] M. Mukherjee et al., ISOLTRAP: An on-line Penning trap for mass spectrometry on short-lived nuclides, Eur. Phys. J. A 35, 1 (2008).

[13] I. Podadera, Ph.D. thesis, Universitat Politècnica de Catalunya, 2006.

[14] J. Dilling et al., Mass measurements on highly charged radioactive ions, a new approach to high precision with TITAN, Int. J. Mass Spectrom. 251, 198 (2006).
[15] F. Herfurth et al., A linear radiofrequency ion trap for accumulation, bunching, and emittance improvement of radioactive ion beams, Nucl. Instrum. Methods Phys. Res., Sect. A 469, 254 (2001).

[16] R. Boussaid, G. Ban, and J. F. Cam, Study of a high intensity radiofrequency cooler, Phys. Rev. ST Accel. Beams 18, 072802 (2015).

[17] R. Boussaid, G. Ban, J. F Cam, and C. Vandamme, Simulations of high intensity ion beam RFQ Cooler for DESIR/ SPIRAL2: SHIRaC, J. Instrum. 9, P07009 (2014).

[18] O. Gianfrancesco, F. Duval, G. Ban, R. B. Moore, and D. Lunney, A radiofrequency quadrupole cooler for highintensity beams, Nucl. Instrum. Methods Phys. Res., Sect. B 266, 4483 (2008).

[19] A. Nieminen, J. Huikari, A. Jokinen, J. Äystö, P. Campbell, and E. C. A. Cochrane, Beam cooler for low-energy radioactive ions, Nucl. Instrum. Methods Phys. Res., Sect. A 469, 244 (2001).

[20] S. Schwarz, G. Bollen, D. Lawton, A. Neudert, R. Ringle, P. Schury, and T. Sun, A second-generation ion beam buncher and cooler, Nucl. Instrum. Methods Phys. Res., Sect. B 204, 474 (2003).

[21] T. Kim, Ph.D. thesis, McGill University, 1997.

[22] D. Manura and D. Dahl, SIMION (R) 8.0 User Manual (2000), http://simion.com/manual/.

[23] Q. Zhao et al., Simulation of solenoid and electrostatic quadrupole focusing of high intensity beam from ECR ion source, in Proceedings of the 21st Particle Accelerator Conference, Knoxville, TN, 2005 (IEEE, Piscataway, NJ, 2005).

[24] M. Berz and K. Makino, MSU Report No. MSUHEP 060803, 2006.

[25] J. Vervier, Status report of EURISOL project, Nucl. Instrum. Methods Phys. Res., Sect. B 204, 759 (2003). 